Relations industrielles

Industrial Relations

\title{
La démocratie industrielle : cogestion ou contrôle ouvrier? Par Dimitri Weiss, Collection relations industrielles, Paris, Les Éditions d'Organisation, 1978, 229 pp.
}

\section{Gérard Dion}

Volume 34, numéro 1, 1979

URI : https://id.erudit.org/iderudit/028946ar

DOI : https://doi.org/10.7202/028946ar

Aller au sommaire du numéro

Éditeur(s)

Département des relations industrielles de l'Université Laval

ISSN

0034-379X (imprimé)

1703-8138 (numérique)

Découvrir la revue

Citer ce compte rendu

Dion, G. (1979). Compte rendu de [La démocratie industrielle : cogestion ou contrôle ouvrier? Par Dimitri Weiss, Collection relations industrielles, Paris, Les Éditions d'Organisation, 1978, 229 pp.] Relations industrielles / Industrial Relations, 34(1), 200-201. https://doi.org/10.7202/028946ar

Tous droits réservés @ Département des relations industrielles de l'Université Laval, 1979
Ce document est protégé par la loi sur le droit d'auteur. L'utilisation des services d'Érudit (y compris la reproduction) est assujettie à sa politique d'utilisation que vous pouvez consulter en ligne.

https://apropos.erudit.org/fr/usagers/politique-dutilisation/ 


\section{RECENSIONS BOOK REVIEWS}

La démocratie industrielle: cogestion ou contrôle ouvrier? par Dimitri Weiss, Collection relations industrielles, Paris, Les Éditions d'Organisation, 1978, $229 \mathrm{pp}$.

Tous ceux qui ont pris connaissance des multiples études de Dimitri Weiss en relations industrielles sont impressionnés à la fois par ses vastes connaissances et par le souci minutieux avec lequel il effectue ses analyses. Peu de chercheurs autant que lui arrivent à se tenir bien informés de tout ce qui se publie dans ce domaine, à bien se l'assimiler et à en faire état dans ses travaux à l'avantage des hommes de sciences et des hommes d'action.

Cet ouvrage est marqué des mêmes caractéristiques et porte sur un sujet d'une grande actualité débattu dans tous les pays. Il est divisé en quatre parties précédées d'une introduction et suivies d'une conclusion. L'auteur a eu la bonne idée d'y joindre une excellente bibliographie ainsi qu'un index.

Dans l'introduction, le professeur Weiss expose les différentes notions de démocratie industrielle et a dit ce qu'il entend par participation en situant celle-ci dans le contexte des pays industrialisés. Il précise aussi l'objet de son ouvrage. «Nous avons souhaité expliquer dans ce livre le concept de démocratie industrielle non seulement au-delà de la cogestion - qui elle, et non la démocratie industrielle dans sa plénitude, cultive ce que l'on pourrait appeler l'" harmonie industrielle» -, mais au-delà de la rhétorique habituelle et des sentences superficielles qui accompagnent trop souvent la simple évocation de ses différentes expressions, en engendrant une confusion regrettable».
Les parties qui suivent répondent parfaitement à ce projet: la cogestion; le contrôle ouvrier; contrôle ouvrier et démocratie syndicale en Italie; une vue comparée; le contrôle ouvrier; quelles perspectives pour la France?

Dans la partie consacrée à la cogestion, l'auteur explique comment on en est venu à un imbroglio sémantique et expose clairement certaines notions fondamentales en apportant les distinctions qui existent entre elles: cogestion et autogestion; codétermination; cogestion et gestion opérationnelle ; la cosurveillance: contrôle, conseil ou orientation?, cosurveillance minoritaire et cosurveillance paritaire; qu'est-ce que la "cogestion paritaire"? la cosurveillance paritaire, partiellement paritaire et quasiparitaire en Allemagne; les réactions à la nouvelle législation allemande en 1976; de la cosurveillance tertiaire à la cosurveillance ternaire: de la parité à la "syndicarchie»? la parité ternaire et la participation externe: le consomméisme. Enfin, l'auteur termine cette partie en se posant quelques questions par rapport à la France.

La seconde partie aborde le sujet du contrôle ouvrier. Ici, encore, le professeur Weiss entreprend un fructueux travail de défrichage entre certains concepts en confrontant à l'occasion la façon dont on les a utilisés. Voici par exemple, quelques soustitre : cogestion et contrôle ouvrier; contrôle préalable au contrôle postérieur; la cogestion paritaire: rupture de l'unité des relations industrielles? contrôle ouvrier et prérogatives managériales; la défense de l'entrepreneurialité"; l'objet du contrôle ouvrier; contrôle ouvrier au contrôle syndical? contrôle ouvrier ou contrôle social? «participation» ou négociation: un débat déjà ancien et toujours actuel ; participation institutionnelle et contrôle confiictuel; 
contrôle ouvrier et autogestion; le contrôle des investissements; la pénétration du syndicat dans le management, etc. Cette simple énumération, incomplète d'ailleurs, en dit déjà long sur la richesse des aspects abordés du contrôle ouvrier.

La troisième partie présente une vue comparée avec le contrôle ouvrier et la démocratie syndicale en Italie. L'auteur qui, depuis plusieurs années, est un observateur perspicace du développement des relations industrielles dans ce pays et qui a déjà consacré plusieurs articles sur le sujet en expose les principales caractéristiques et n'est pas loin de penser que ce pays peut être le laboratoire social de l'Europe.

L'étude du professeur Weiss nous repose des ouvrages doctrinaires où les préoccupations de promouvoir une idéologie dispensent souvent de s'arrêter à bien saisir les complexités de la vie réelle. Elle contribuera à faire avancer la démocratie industrielle beaucoup plus que toute la rhétorique romantique qui lui a été consacrée jusqu'ici.

Nous croyons que désormais, si l'on veut être sérieux, on ne pourra plus s'aventurer à parler de démocratie industrielle, de cogestion, d'autogestion ou de contrôle ouvrier sans se référer à l'ouvrage du professeur Weiss.

Gérad Dion

Université Laval

Gestion des ressources humaines. Une approche systémique, par Laurent Bélanger, Chicoutimi, Gaétan Morin et Associés Limitée, 1978, 363 pp.

L'ouvrage de Laurent Bélanger vise à présenter une reformulation globale et intégrée de la gestion des ressources humaines. Le défi est de taille mais l'auteur le relève d'une façon originale et utile. En effet, plusieurs groupes de lecteurs trouveront grand intérêt à lire ou à consulter cet ouvrage. L'étudiant de niveau universitaire, le praticien qui débute sa carrière, le professionnel de la gestion des ressources humaines, etc. trouveront, du moins l'auteur le souhaite, «à repenser et à réorien- ter sa pratique quotidienne». Il faut avouer que cet objectif est atteint du moins dans la première partie de l'œuvre de Laurent Bélanger qui traite de la dimension administrative de la gestion des ressources humaines.

L'auteur qui s'appuie en grande partie sur l'esprit des sciences du comportement et de la théorie des systèmes, divise son ouvrage en deux grandes parties. D'une part, il consacre huit (8) exposés ou chapitres à la description des activités de nature administrative normalement accomplies par un service de gestion des ressources humaines. D'autre part, il expose ses vues sur la notion de climat organisationnel, la création d'un milieu de travail satisfaisant et valorisant ainsi que sur certains problèmes qui, lorsqu'ils sont amoindris, permettent l'existence d'une meilleure qualité de vie au travail.

Une première constatation qui frappe le lecteur est la disposition qui existe entre les partie I et II de l'ouvrage de Laurent Bélanger. Bien que l'auteur considère que la gestion des ressources humaines comprend deux (2) grandes dimensions soit la dimension opérationnelle (activités reliées à l'acquisition, au maintien et au développement des ressources humaines) et la dimension énergétique (activités reliées à la création et au maintien d'un climat organisationnel ou d'un milieu de travail satisfaisant, voir même valorisant pour les individus), il excelle beaucoup plus dans sa présentation reliée à la dimension opérationnelle. Il est un peu malheureux qu'il en soit ainsi car la dimension énergétique de la gestion des ressources humaines est celle qui fait actuellement surface en matière de gestion des ressources humaines et c'est celle qui cause le plus de difficultés à ceux qui ont pour travail d'améliorer la qualité de vie au travail.

Dans sa première partie, l'auteur (après avoir présenté un excellent chapitre à la vision systémique de la gestion des ressources humaines) présente d'une façon très logique les principales dimensions opérationnelles de la gestion des ressources humaines. Plusieurs thèmes $\mathrm{y}$ sont présentés d'une façon claire et avec un bon dosage d'exhaustivité. Le support structural de la gestion des ressources humaines 